\title{
Individual differences in syntactic ambiguity resolution: Readers vary in their use of plausibility information
}

\author{
Debra L. LoNG \\ University of California, Davis, California \\ and University of Central Lancashire, Preston, England \\ AND \\ Chantel S. Prat \\ Carnegie Mellon University, Pittsburgh, Pennsylvania
}

\begin{abstract}
Two experiments investigated the relation between individual differences in working memory capacity and differences in the efficiency of syntactic processing. In one experiment, readers comprehended sentences containing main-verb/reduced-relative ambiguities that all resolved to the reduced-relative interpretation. High-span (but not low-span) readers processed sentences more slowly when the sentences were biased to the preferred, main-verb interpretation than when they were biased to the reduced-relative interpretation. Moreover, high-span (but not lowspan) readers used information about the plausibility of the different interpretations even though low-span readers appeared to possess the requisite knowledge. In Experiment 2, readers received intensive exposure to sentences with main-verb/reduced-relative ambiguities. Exposure enhanced low-span readers' use of plausibility information. Moreover, the effect of exposure generalized to sentences that were not included in the training materials.
\end{abstract}

Reading is a complex skill consisting of multiple component processes. The ability to learn and execute these processes is variable; large individual differences in reading comprehension are found even among college students (Bell \& Perfetti, 1994; Gernsbacher \& Faust, 1991, 1995; Long, Oppy, \& Seely, 1994, 1997; Stanovich \& West, 1989). Research has identified some of the characteristics that predict reading proficiency, such as word recognition skill, working memory capacity, and domain knowledge. Reader characteristics alone, however, cannot explain the variability in comprehension performance because comprehension depends critically on the nature of the material that is read (e.g., complexity of the language, genre of the text). Individuals and materials interact in all aspects of language processing. In this study, we examined one such interaction - the interaction between an individual's working memory capacity and the syntactic complexity of a sentence.

Several studies have reported a correlation between working memory capacity - as measured by performance on the reading-span task - and the speed and accuracy of syntactic processing (Just \& Carpenter, 1992; King \& Just, 1991; MacDonald, Just, \& Carpenter, 1992; Pearlmutter \& MacDonald, 1995). This relation is important because it has been used as evidence in two debates. One concerns theories of syntactic processing and predictions about when and how contextual information is used in ambiguity resolution. The second debate is the focus of this study; it concerns the nature of working memory limitations and predictions about why variation in capacity affects syntactic processing. In the next section, we describe the sources of information that are used in resolving syntactic ambiguities. We then describe three views of working memory capacity and how capacity is related to individual variation in syntactic analysis.

\section{Syntactic Ambiguity Resolution}

A sentence is syntactically ambiguous when a sequence of words is compatible with more than one grammatical structure. Ambiguity can be global in that an entire sentence has more than one interpretation (e.g., They are cooking apples) or it can be local in that a sequence of words is compatible with more than one interpretation until additional information is encountered (e.g., The horse raced past the barn fell).

Many studies of syntactic analysis have focused on main-verb/reduced-relative ambiguities, such as The defendant examined by the lawyer shocked the jury. The first verb (examined) has at least two interpretations: It may be the main verb of the sentence (e.g., The defendant examined the jury's faces) or it may introduce a reducedrelative clause. Main-verb structures appear more often in language than do reduced-relative ones; thus, readers are biased to adopt the main-verb interpretation (Bever, 1970). This bias can lead to momentary difficulty when they en- 
counter information that is inconsistent with the preferred interpretation. Researchers have identified many sources of information that can be used to resolve such ambiguities. Two of these are important in the present study.

1. Verb frequency information. Two sources of frequency information can influence how readers process main-verb/ reduced-relative ambiguities (MacDonald, Pearlmutter, \& Seidenberg, 1994; McRae, Spivey-Knowlton, \& Tanenhaus, 1998; Trueswell, 1996). First, verbs vary in the frequency with which they appear in their past-tense and past-participle forms. This variation is important because it relates to the distinction between active and passive structures. The main-verb structure is active and requires the past-tense form, whereas the reduced-relative interpretation is passive and requires the past-participle form. Second, verbs vary in how often they appear in transitive and intransitive structures. The reduced-relative structure requires a verb that takes a direct object (transitive), whereas the main-verb structure can appear with both transitive and intransitive verbs (Hare, Tanenhaus, \& McRae, 2007; Trueswell, 1996). Thus, main-verb/reduced-relative structures are difficult to process when the ambiguous verb appears frequently in its past-tense/active/transitive form and a reduced-relative interpretation is required (Trueswell, Tanenhaus, \& Garnsey, 1994).

2. Plausibility of the initial noun phrase as agent or patient. Some noun phrases are more plausible than others as the agents of a verb. Animacy is one factor that influences plausibility because it correlates with sentence structure. Animate noun phrases are likely to be agents in main-verb structures (e.g., The witness examined by the lawyer was unreliable) and inanimate noun phrases are likely to be patients in reduced-relative ones (e.g., The evidence examined by the lawyer was unreliable). Plausibility is also influenced by world knowledge. For example, the noun phrase the cops is a more plausible agent of the verb arrested (e.g., The cops arrested the men) than is the phrase the criminals (e.g., The criminals arrested the $m e n)$. Readers have more difficulty with reduced-relative interpretations when noun phrases are plausible agents (e.g., The cops arrested by the FBI were ... .) than when they are less plausible (e.g., The criminals arrested by the FBI were ...) (McRae, Ferretti, \& Amyote, 1997; McRae et al., 1998; Pearlmutter \& MacDonald, 1995; Tabossi, Spivey-Knowlton, McRae, \& Tanenhaus, 1994).

Theories of syntactic parsing are generally similar with respect to claims about the sources of information that are used in ambiguity resolution, but differ about when they are used. Syntactic theories can be categorized into two classes: two-stage and constraint-based. The most wellknown of the two-stage accounts is the garden path model proposed by Frazier and colleagues (Frazier, 1987; Frazier \& Clifton, 1996). According to the model, syntactic analysis proceeds in two stages. First, a syntactic interpretation system constructs a structure for each sentence on the basis of the lexical categories of the input (e.g., verb, noun), the syntactic rules of the language, and the preference heuristics that bias interpretation to some structures more than to others (e.g., minimal attachment). Second, semantic and contextual information is integrated with the structure that was developed in the first stage. When contextual information is inconsistent with the initial structure, revision may be triggered and a new contextually consistent structure may be developed.

An alternative view of syntactic analysis is found in the constraint-based model proposed by MacDonald et al. (1994). According to this model, syntactic structure is developed on the basis of the operation of numerous combinatorial constraints that occur at both lexical and syntactic levels. When readers encounter a word, word meanings and fragments of syntactic structure associated with the word compete for activation in a distributed network. Competition is influenced by multiple sources of information, including context. The outcome of the competitive process is a word meaning and a syntactic role that become integrated into the developing representation.

Both the garden path and constraint-based models claim that contextual information is used to resolve syntactic ambiguities. They are distinguished primarily by their claims about when the information is used. Contextual information has an earlier effect in the constraint-based model than it does in the garden path model.

Our focus in this study was on individual variation in the use of probabilistic information for resolving syntactic ambiguities rather than on claims about when the information is used. We compared reading times for ambiguous sentences that were biased to a main-verb interpretation to those for ambiguous sentences that were biased to a reduced-relative interpretation. Thus, we did not have the unambiguous baseline condition that would allow us to distinguish between the garden path and constraint-based models.

\section{Nature of Working Memory Limitations in Sentence Processing}

A central thesis of most working memory models is that individuals vary in capacity. In this section, we describe three views of capacity limitations that make predictions about how working memory relates to syntactic analysis. Just and Carpenter (1992) have presented one view called capacity theory, which claims that the storage and processing functions that are necessary for language are fueled by activation, a commodity that maintains knowledge elements in memory and supports computation. Activation is shared among storage and processing functions; activation-consuming processes limit the activation available to support storage. Capacity theory attributes variation in language processing, including syntactic analysis, to differences in capacity- the amount of activation available to the system.

A second view of working memory limitations emphasizes the roles of practice and skill in comprehension. This view is represented in the distributed-learning model proposed by MacDonald and Christiansen (2002). Variation in practice can lead to individual differences that may appear qualitative. For example, reduced-relative structures have a low frequency in language. Low-span readers are likely to encounter them infrequently; thus, according to the distributed-learning model, these structures should be difficult for them to understand. Moreover, they should 
have particular difficulty understanding object-relative clauses because of their low-frequency word order (i.e., noun, noun, verb).

A third view of working memory limitations is the separate sentence interpretation resource theory proposed by Caplan and Waters (1999). According to their model, verbal tasks are supported by two pools of processing resources: a specialized pool and a general one. The specialized pool supports the initial interpretation of sentence meaning, including the assignment of syntactic structure. The general pool is akin to the domain-general activation pool described in capacity theory. This pool supports the use of sentence meaning to accomplish tasks such as recall and meaning judgment. Individual variation is found only in the general pool. Readers do not differ in the specialized system that is responsible for the initial processing of a sentence.

\section{Relation Between Working Memory Capacity and Syntactic Analysis}

Studies that examine the relation between working memory capacity and syntactic analysis are marked by numerous inconsistencies, which often arise from differences in the methods and materials used. King and Just (1991) were the first to document this relation. They found that lowspan readers were slower and less accurate than high-span readers in processing object-relative sentences such as The senator that the reporter attacked admitted the error. King and Just argued that object-relative clauses were demanding for the low-span readers because these readers did not have the capacity to assign two syntactic roles (e.g., subject and object) to a single entity (e.g., reporter).

MacDonald et al. (1992) also found a relation between span and syntactic ambiguity resolution. In their study, high-span readers showed slower processing of syntactically ambiguous sentences relative to unambiguous ones than did low-span readers. Readers received sentences in which the information that followed an initial noun phrase was temporarily ambiguous (e.g., The soldiers warned about the dangers . . .). High-span readers were slower than low-span readers to process ambiguous sentences that resolved to either a main-verb interpretation (e.g., The experienced soldiers warned about the dangers before the midnight raid) or a reduced-relative interpretation (e.g., The experienced soldiers warned about the dangers conducted the midnight raid). MacDonald et al. (1992) argued that high-span readers had sufficient capacity to maintain multiple interpretations until they reached the disambiguating information. These multiple interpretations imposed a processing cost, however, leading to slower reading times in the ambiguous region for highspan than for low-span readers.

Pearlmutter and MacDonald (1995) examined reading times for a prepositional phrase that followed a syntactically ambiguous verb (e.g., The soup cooked in the pot but was not ready to eat). High-span readers processed words that conveyed disambiguating information (i.e., the preposition) more slowly than did low-span readers, a result that is similar to the pattern reported by MacDonald et al. (1992). In a series of regression analyses, Pearlmutter and MacDonald showed that the source of the reading-time differences was the variation in readers' use of probabilistic constraints - in particular, the use of plausibility information about alternative interpretations.

Waters and Caplan (1996) examined sentences containing temporary syntactic ambiguities, similar to those used by MacDonald et al. (1992). Waters and Caplan found that ambiguous sentences were more difficult to comprehend than were unambiguous ones, but they found no span differences (see also Clifton et al., 2003). Similarly, Caplan and Waters (1999) found no span differences in the processing of object-relative sentences. They concluded that capacity differences do not give rise to individual variation in the syntactic interpretation of sentences.

Our goal in this study was to explore further the relation between individual differences in working memory capacity and syntactic processing. Our study differs from those conducted previously in that we used a much larger number of sentences containing syntactically complex structures than has been used before. In Experiments 1A, 1B, and $1 \mathrm{C}$, we had readers comprehend sentences containing main-verb/reduced-relative structures and manipulated the task associated with reading. In Experiment 1A, participants read the sentences and only answered questions that followed the filler sentences. We did not ask questions about the ambiguous sentences in order to address the concern that directing comprehension questions at the ambiguous sentences might alert the high-span, highverbal participants to the nature of the experimental manipulation (Waters \& Caplan, 1996; but see MacDonald \& Christiansen, 2002, for a response to this concern).

Experiments 1B and 1C were conducted to determine whether low-span readers eventually arrive at the correct interpretation of the main-verb/reduced-relative ambiguities. In Experiment 1B, we asked comprehension questions about the ambiguous sentences and examined accuracy as a function of span. We conducted a third experiment to address our concern that many of the comprehension questions in Experiment 1B could be answered correctly on the basis of world knowledge and a shallow understanding of the sentence. Thus, Experiment 1C included a grammaticality judgment task to determine whether high-span and low-span readers differed in their judgments about the acceptability of the sentences.

One drawback of using a large number of sentences containing main-verb/reduced-relative ambiguities was that the sentences were very heterogeneous with respect to properties that may influence ambiguity resolution (e.g., frequency, plausibility). An advantage of this heterogeneity, however, was that it allowed us to conduct a series of regression analyses to examine variation in readers' sensitivity to these properties, analyses similar to those conducted previously by Pearlmutter and MacDonald (1995).

Our second aim in this study was to determine whether differences in readers' sensitivity to probabilistic information in resolving main-verb/reduced-relative ambiguities would be affected by repeated exposure to these structures. In Experiment 2, readers were exposed to large numbers of main-verb/reduced-relative sentences. Our goal was to determine whether repeated experience would influence readers' use of probabilistic information during reading. 


\section{EXPERIMENT 1}

Our goals in Experiment 1 were (1) to examine the influence of working memory capacity on the processing of syntactically ambiguous sentences (Experiment 1A), (2) to determine whether reading-time differences as a function of span were related to low-span readers' failure to construct the reduced-relative interpretation (Experiments 1B and $1 \mathrm{C}$ ), and (3) to examine readers' sensitivity to probabilistic information in the sentences (regression analyses).

Our procedure in Experiments 1A, 1B, and 1C was similar to that used in many previous studies of syntactic ambiguity. We had participants read sentences that were presented one word at a time in a moving-window paradigm. Each sentence began with a noun phrase and then a verb that was part of a reduced-relative clause. The verb was followed by a prepositional phrase and then the main verb of the sentence. All our experimental sentences resolved to a reduced-relative interpretation (past-participle morphology, passive voice, transitive argument structure).

Each of our experimental sentences had two versions. In one version, the verb in the relative clause was more frequent in its past-tense form than in its past-participle form (past-tense-biased condition). A second version of the sentence was created by replacing the past-tense-biased verb with one that was biased to its past-participle form (pastparticiple-biased condition). The set of verbs in this condition included some that were unambiguously past participle (i.e., morphologically irregular verbs, such as eaten).

\section{Experiment 1A}

We examined reading times for an ambiguity effect at two regions in the sentence: at the prepositional phrase (when the main-verb interpretation became less likely) and at the remainder of the sentence (when the reducedrelative interpretation was favored). Questions about the sentences were included to encourage comprehension, and those after filler sentences were included for the reason described above.

We also had three groups of participants read the experimental sentences and make judgments about them. One group was asked to rate the plausibility of the initial noun phrase as a theme of the verb in the relative clause. A second group was asked to rate the plausibility of the initial noun phrase as the agent. A third group was asked to produce completions to sentence fragments containing the initial noun phrase and the verb. All the participants received the Daneman and Carpenter (1980) reading-span task.

Both capacity theory and the distributed-learning model predict differences in ambiguity resolution as a function of working memory capacity. Specifically, they predict that high-span readers should process the prepositional phrase following the verb in the reduced-relative clause more slowly than should low-span readers. Capacity theory bases this prediction on the claim that highspan readers can hold multiple interpretations in memory simultaneously, which imposes a processing cost. The distributed-learning model makes the prediction based on the claim that high-span readers have more experience with low-frequency structures than do low-span readers.
In contrast, the separate sentence interpretation resource theory predicts no differences in ambiguity resolution as a function of span, particularly in the absence of comprehension questions that might alert high-span readers to the nature of the sentences.

\section{Method}

Participants. Participants were 222 undergraduate students at the University of California, Davis. Forty-two students participated in the reading-time study; the remaining students performed rating and sentence-completion tasks. All participants spoke English as their first language, and none had a diagnosed reading disability. The students received course credit for their participation.

Materials. We constructed 120 experimental sentences; each sentence had a past-tense-biased and a past-participle-biased version. The list of sentences appears in the Appendix. Table 1 contains descriptive information about the sentences. The past-tense-biased versions were similar to the following: The salad tossed for the party looked delicious. The sentence began with an inanimate noun phrase and was followed by a verb that was ambiguous between its pasttense and past-participle forms. The past-tense form was always the more frequent one, as measured using frequency counts in the Penn Treebank Corpus (Marcus, Marcinkiewicz, \& Santorini, 1993). The verb was followed by a three-word prepositional phrase. The prepositional phrases played various semantic roles in the sentences (locative, $48 \%$; temporal, 23\%; direction, 20\%; purpose or reason, $6 \%$; and manner, $4 \%$ ); however, the semantic role was always the same in the two versions of the sentence. The prepositional phrase was followed by a verb that disambiguated the reduced-relative interpretation.

The past-participle-biased versions followed the same format as the past-tense-biased ones, except that the verb in the relative clause was either unambiguously past participle (e.g., eaten, bitten; 37\%) or appeared more frequently in its past-participle than its past-tense form (e.g., The salad mixed for the party looked delicious). The verbs also differed in transitivity. Sixty-eight percent of the verbs in the past-tense-biased version had both transitive and intransitive interpretations, whereas $28 \%$ of the verbs in the past-participle-biased condition had both interpretations.

We also constructed 120 filler sentences. These sentences had a variety of syntactic forms. Sixty percent were canonical, nounverb-noun sentences; the remainder contained relative clauses. However, the relative clauses in the filler sentences were unambiguously signaled by the appropriate function words (e.g., that was/that were). The filler sentences were similar to the experimental ones in that they all began with inanimate noun phrases.

Test questions were included to encourage participants to read for comprehension. The questions consisted of 48 paraphrases of preceding filler sentences. Half the sentences were true paraphrases, and half were false.

Two lists of stimulus sentences were constructed. Each contained 240 sentences: 120 experimental sentences (60 in the past-tensebiased and 60 in the past-participle-biased condition) and 120 filler sentences. The two versions of each sentence (past-tense biased and past-participle biased) were counterbalanced across material sets.

Table 1

Verb Frequency in the Experimental Sentences As a Function of Verb Bias

\begin{tabular}{|c|c|c|c|c|}
\hline \multirow[b]{3}{*}{ Log Frequency } & \multicolumn{4}{|c|}{ Verb-Bias Condition } \\
\hline & \multicolumn{2}{|c|}{$\begin{array}{c}\text { Past-Tense } \\
\text { Bias }\end{array}$} & \multicolumn{2}{|c|}{$\begin{array}{c}\text { Past-Participle } \\
\text { Bias }\end{array}$} \\
\hline & $M$ & $S D$ & $M$ & $S D$ \\
\hline Past tense & 7.35 & 1.71 & 4.06 & 1.90 \\
\hline Past participle & 4.86 & 1.60 & 6.81 & 1.53 \\
\hline Base & 7.49 & 1.92 & 7.61 & 1.68 \\
\hline
\end{tabular}


Procedure. The experiment was conducted in two phases. The Daneman and Carpenter (1980) reading-span task was administered in the first phase. These sessions occurred over a 3-day period. Participants read aloud a set of unrelated sentences, which were presented one at a time, and recalled the final word of each sentence once the entire set had been presented. The number of sentences in a set varied from two to six. A participant's reading span was calculated as the total number of sentence-final words recalled. ${ }^{1}$

The second phase occurred approximately $24 \mathrm{~h}$ after the last reading-span session. Participants were assigned to one of the two material sets. We used the following procedure to ensure that we had similar span distributions in each counterbalancing condition. We scored the reading-span test and ranked all participants according to their performance. We proceeded through the ranking, selecting pairs of participants. The first member of each pair was randomly assigned to one counterbalancing condition; the second member of the pair was assigned to the other condition.

The stimulus sentences were presented randomly using a movingwindow display. Each sentence was preceded by an asterisk that appeared for 1,000 msec. Participants read the sentence by pressing the space bar. Each keypress revealed a word. Filler sentences were followed by comprehension questions. Participants were told to read the question and to determine whether it was true or false about the immediately preceding sentence. They indicated their response by pressing one of two keys labeled true and false.

The remaining participants were also tested in two phases. They received the reading-span task and then performed a rating or a sentence completion task. The procedure for assigning participants to counterbalancing conditions was the same as that described above. One group $(n=60)$ provided ratings about the plausibility of the initial noun phrase being the theme of the verb in the relative clause. The materials for these ratings consisted of sentence fragments that ended after the verb in the relative clause and before the prepositional phrase. We were interested in participants' ratings of the relative-clause interpretation; thus, the sentence fragments contained unreduced relative clauses, constructed by inserting that was/ that were into the reduced-relative fragments (e.g., The salad that was tossed ...). Participants were asked to rate the plausibility of the event in the fragment on a 7-point scale from 1 (not at all plausible) to 7 (highly plausible).

A second group of participants $(n=60)$ provided ratings about the plausibility of the initial noun phrase as the agent of the verb in the relative clause. The materials for these ratings consisted of sentence fragments that ended after the verb in the relative clause. We were interested in participants' ratings of the main-verb interpretation; thus, the sentence fragments contained the initial noun phrase followed by the verb (e.g., The salad tossed . . .). The verb was always presented in its past tense form. Participants were asked to rate the plausibility of the event in the fragment on a 7-point scale from 1 (not at all plausible) to 7 (highly plausible).

The final group $(n=60)$ provided completions for the sentences. They received fragments consisting of the initial noun phrase followed by the verb (e.g., The salad tossed . . .). Participants were asked to generate a sensible completion for each fragment.

\section{Results and Discussion}

Rating and completion data. Participants who provided rating and completion data were classified into high-span, medium-span, and low-span groups based on their recall of words in the reading-span task. The themeplausibility group had mean span scores of $49.9,41.3$, and 29.4 for high-span, medium-span, and low-span readers, respectively. The agent plausibility group had scores of $51.4,42.4$, and 32.1 for high-span, medium-span, and low-span readers, respectively. The sentence-completion group had scores of 52.1, 44.3, and 30.9 for high-span, medium-span, and low-span readers, respectively.
We computed mean ratings for the theme- and the agentplausibility data as a function of verb bias (past-tense biased, past-participle biased) and reading span. The sentencecompletion data were scored as the proportion of participants who completed the sentence with a relative clause. Means as a function of reading span and verb bias appear in Table 2.

We performed separate 2 (verb bias) $\times 3$ (span) ANOVAs on the theme-rating, agent-rating, and sentencecompletion data. As can be seen in Table 2, participants found the nouns to be relatively implausible agents of the verbs in the relative clauses. This did not differ as a function of verb bias or reading span (all $F \mathbf{s}<1$ ). In contrast, participants found that the nouns were relatively plausible themes of the verbs. Again, this did not differ by verb bias or reading span (all $F_{\mathbf{S}}<1$ ). We did find a reliable effect of verb bias in our analysis of the sentence-completion data. Sentence fragments in the past-participle-biased condition received more relative-clause continuations than did those in the past-tense-biased condition $[F(1,119)=14.87$, $\left.M S_{\mathrm{e}}=0.02\right]$. We found no other reliable effects $(F \mathrm{~s}<1)$.

Reading-time analyses. We calculated reading times for two regions in the sentences. The first region (prepositional phrase) consisted of a three-word prepositional phrase; the second region (sentence final) consisted of the remaining words in the sentence. Reading times more than 3 standard deviations from a participant's mean were treated as missing data and excluded from the analysis $(0.9 \%$ of the data). All effects were tested with an alpha level of .05; effect sizes are indicated by Cohen's $d$. Participants were classified into one of three groups as described above $(M \mathrm{~s}=51.5,44.2$, and 31.7 for high span, medium span, and low span, respectively).

We performed 2 (verb bias) $\times 3$ (span) ANOVAs on reading times for the two sentence regions. The means appear in Table 3. Our analysis at the prepositional phrase yielded a reliable effect of verb bias $\left[F_{1}(1,39)=5.31\right.$, $\left.M S_{\mathrm{e}}=17,529 ; F_{2}(1,177)=7.49, M S_{\mathrm{e}}=49,323\right]$. We also found a main effect of span, which was reliable in the item analysis $\left[F_{2}(2,177)=25.05, M S_{\mathrm{e}}=121,146\right]$ but not in the participant analysis $\left[F_{1}=1.65, M S_{\mathrm{e}}=431,787\right.$, $p=.21]$. These effects were modified by the criticalverb bias $\times$ span interaction $\left[F_{1}(2,39)=4.26, M S_{\mathrm{e}}=\right.$ 17,$\left.529 ; F_{2}(2,177)=6.61, M S_{\mathrm{e}}=49,323\right]$. High-span readers were slower to read the prepositional phrase in past-tense-biased than in past-participle-biased sentences

Table 2

Mean Plausibility Ratings and Sentence Completions As a Function of Verb Bias and Reading Span

\begin{tabular}{lccc}
\hline & \multicolumn{3}{c}{ Reading Span } \\
\cline { 2 - 4 } Verb Bias & High & Medium & Low \\
\hline Agent Plausibility & & & \\
$\quad$ Past tense & 2.64 & 2.19 & 2.88 \\
$\quad$ Past participle & 2.77 & 2.91 & 2.93 \\
Theme Plausibility & & & \\
$\quad$ Past tense & 4.23 & 4.99 & 4.46 \\
$\quad$ Past participle & 4.84 & 4.52 & 4.35 \\
Relative Clause Continuation & & & \\
$\quad$ Past tense & .24 & .21 & .24 \\
Past participle & .34 & .31 & .28 \\
\hline
\end{tabular}


$\left[F_{1}(2,39)=13.79, M S_{\mathrm{e}}=17,529, d=0.14 ; F_{2}(2,177)=\right.$ $\left.20.67, M S_{\mathrm{e}}=49,323\right]$, whereas medium-span and lowspan readers showed no reliable RT differences $(F \mathrm{~s}<1)$.

Our analyses of reading times in the sentence-final region revealed a reliable effect of verb bias $\left[F_{1}(1,39)=\right.$ $\left.40.80, M S_{\mathrm{e}}=14,913 ; F_{2}(1,177)=51.81, M S_{\mathrm{e}}=426,359\right]$. Participants exhibited slower reading times for past-tensebiased than for past-participle-biased sentences $(d=0.27)$. This pattern did not differ as a function of $\operatorname{span}\left(F_{\mathrm{S}}<1\right)$.

Our results suggest that readers, regardless of span, had more difficulty comprehending sentences that were biased to the main-verb than they did those that were biased to the reduced-relative interpretation. High-span readers showed the effect of verb bias early in the sentence, at the prepositional phrase. Medium-span and low-span readers, however, did not show an ambiguity effect until later in the sentence, after encountering the main verb.

We did not include comprehension questions about the experimental items in this study so that we could examine the claim that questions alert high-span readers to the nature of the stimuli. In Experiment 1B, we added questions after each experimental sentence to assess the extent to which readers constructed the correct syntactic interpretation of the reduced-relative sentences.

\section{Experiment 1B}

Experiment 1B was a replication of the previous experiment except that each experimental item was followed by a comprehension question. Our analyses of the readingtime data included only sentences for which readers made a correct response.

\section{Method}

Participants. Participants were 48 undergraduate students at the University of California, Davis. All spoke English as their first language, and none had a diagnosed reading disability. The students received course credit for their participation. Participants were classified into three groups, as in Experiment 1A: high span $(M=50.5)$, medium span $(M=41.7)$, and low span $(M=36.8)$.

Materials and Procedure. The experimental and filler sentences were the same as those used in the previous experiment. We wrote additional questions to assess the participants' comprehension of the experimental items. The questions were paraphrases that targeted the role of the initial noun phrase in the relative clause. True paraphrases included the initial noun phrase, the verb from the relative clause, and the prepositional phrase (e.g., The salad was mixed for the party). False paraphrases contained the initial noun phrase, but the verb from the relative clause or the prepositional phrase was different from the original (e.g., The salad was bought for the party/ The salad was mixed in the kitchen). The procedure was the same as that used in Experiment $1 \mathrm{~A}$.

\section{Results and Discussion}

We analyzed the data as described previously. We excluded reading times more than 3 standard deviations from a participant's mean (1.5\% of the data). We also identified sentences that were associated with incorrect responses on the comprehension questions and excluded the reading times for those sentences (19.5\% of the data).

Comprehension questions. High-span and mediumspan readers were more accurate than low-span readers, but not reliably so $(M \mathrm{~s}=81.7 \%, 82.0 \%$, and $79.2 \%$ for high
Table 3

Reading Times (in Milliseconds) for the Prepositional-Phrase and Sentence-Final Regions As a Function of Verb Bias and Reading Span in Experiment 1A

\begin{tabular}{|c|c|c|c|c|c|c|}
\hline \multirow[b]{3}{*}{ Region Bias } & \multicolumn{6}{|c|}{ Reading Span } \\
\hline & \multicolumn{2}{|c|}{ High } & \multicolumn{2}{|c|}{ Medium } & \multicolumn{2}{|c|}{ Low } \\
\hline & $M$ & $S D$ & $M$ & $S D$ & $M$ & $S D$ \\
\hline \multicolumn{7}{|c|}{ Prepositional Phrase } \\
\hline Past tense & 1,274 & 700 & 984 & 308 & 1,043 & 440 \\
\hline Past participle & 1,189 & 522 & 980 & 314 & 1,032 & 407 \\
\hline \multicolumn{7}{|l|}{ Sentence Final } \\
\hline Past tense & 1,481 & 469 & 1,585 & 551 & 1,580 & 514 \\
\hline Past participle & 1,355 & 399 & 1,460 & 528 & 1,443 & 467 \\
\hline
\end{tabular}

span, medium span, and low span, respectively). We found no reliable effects involving verb bias or span $(F \mathrm{~s}<1)$.

Reading-time analyses. We performed 2 (verb bias) $\times 3$ (span) ANOVAs on the mean reading times for the two sentence regions. The means appear in Table 4. Our analyses of the data for the prepositional phrase yielded a reliable effect of verb bias in the participant analysis $\left[F_{1}(1,45)=5.80, M S_{\mathrm{e}}=5,024\right]$ but not in the item analysis $\left[F_{2}(1,177)=1.24, M S_{\mathrm{e}}=38,200\right]$. This was modified by a verb bias $\times$ span interaction $\left[F_{1}(2,45)=9.15, M S_{\mathrm{e}}=\right.$ $5,024, p=.26]$ that was reliable in the participant analysis and marginally reliable in the item analysis $\left[F_{2}(2,177)=\right.$ $\left.2.59, M S_{\mathrm{e}}=38,200, p=.07\right]$. High-span readers were slower in the past-tense-biased condition than they were in the past-participle-biased condition $\left[F_{1}(2,45)=23.80\right.$, $\left.M S_{\mathrm{e}}=5,024, d=0.40 ; F_{2}(2,177)=6.24, M S_{\mathrm{e}}=38,200\right]$, whereas medium-span and low-span readers showed no reliable differences $(F \mathrm{~s}<1)$.

In the sentence-final region, we found a reliable effect of verb bias $\left[F_{1}(2,45)=264.42, M S_{\mathrm{e}}=4,697 ; F_{2}(2,177)=\right.$ $\left.56.64, M S_{\mathrm{e}}=426,332\right]$. Participants' reading times were faster in the past-participle-biased condition than they were in the past-tense-biased condition $(d=0.28)$. No other effects were reliable (all $F \mathrm{~s}<1$ ).

The results of Experiment 1B are consistent with those of the previous experiment. High-span readers comprehended the prepositional phrase more slowly in the pasttense-biased condition than they did in the past-participlebiased condition in both experiments, whereas all readers showed the verb-bias effect at the end of the sentence. This pattern occurred irrespective of the nature of the comprehension questions.

Table 4

Reading Times (in Milliseconds) for the Prepositional-Phrase and Sentence-Final Regions As a Function of Verb Bias and Reading Span in Experiment 1B

\begin{tabular}{|c|c|c|c|c|c|c|}
\hline \multirow[b]{3}{*}{ Region Bias } & \multicolumn{6}{|c|}{ Reading Span } \\
\hline & \multicolumn{2}{|c|}{ High } & \multicolumn{2}{|c|}{ Medium } & \multicolumn{2}{|c|}{ Low } \\
\hline & $M$ & $S D$ & $M$ & $S D$ & $M$ & $S D$ \\
\hline \multicolumn{7}{|c|}{ Prepositional Phrase } \\
\hline Past tense & 1,138 & 347 & 1,019 & 368 & 987 & 406 \\
\hline Past participle & 1,016 & 250 & 985 & 340 & 974 & 375 \\
\hline \multicolumn{7}{|l|}{ Sentence Final } \\
\hline Past tense & 1,479 & 366 & 1,491 & 548 & 1,514 & 538 \\
\hline Past participle & 1,361 & 321 & 1,357 & 501 & 1,386 & 486 \\
\hline
\end{tabular}




\section{Experiment 1C}

The results of Experiment 1B show that high-span and low-span readers were equally accurate in answering questions about information in the relative clause. These results are not strong evidence, however, that readers successfully resolved the main-verb/reduced-relative ambiguity. Consider the following sentence: The first question asked/written on the exam was surprisingly difficult. A comprehension question about the role of the initial noun phrase (e.g., The question was asked on the exam) can be answered easily on the basis of world knowledge and a relatively superficial understanding of the sentence. Given the nature of the sentences, we thought it unlikely that comprehension questions could be written in a way that would minimize this concern. Thus, we addressed the issue using a different approach. Rather than ask readers comprehension questions, we had them make grammaticality judgments. This decision was based on the hypothesis that readers who failed to arrive at a correct interpretation of the syntactic ambiguity would be more likely than other readers to judge the sentence as ungrammatical. Thus, we had participants make a grammaticality judgment after each experimental and filler sentence. New filler sentences were written such that each contained a grammatical error. The nature of the errors is described below.

\section{Method}

Participants. Participants were 48 undergraduate students at the University of California, Davis. All spoke English as their first language, and none had a diagnosed reading disability. The students received course credit for their participation. Participants were classified into three groups, as in Experiments 1A and 1B: high span $(M=48.7)$, medium span $(M=39.2)$, and low span $(M=34.5)$.

Materials and Procedure. The experimental sentences were the same as those used in the previous experiments. We wrote new filler sentences that were similar to the experimental ones. The fillers began with an initial noun phrase (inanimate), followed by a relative clause, a prepositional phase, and the main verb. In $40 \%$ of the sentences, the relative clause was preceded by appropriate function words. We varied the nature of the grammatical violations in the sentences. These included number violations in the relative clause (e.g., The cookies that was baked in the oven were delicious), number violations in the main clause (e.g., The box hidden in the closet were full of pictures), inappropriate prepositions in the prepositional phrase (e.g., The chairs that were stacked of the wall fell to the ground), animacy violations (e.g., The eggs that were tossed on the counter leapt to the floor), and transitivity violations (e.g., The soup bubbled in the pot was delicious).

The procedure was the same as that used in Experiments 1A and $1 \mathrm{~B}$, except that each experimental and filler sentence was followed by a grammaticality judgment. Participants were asked to make a yes/no decision as to whether or not the immediately preceding sentence was grammatically correct.

\section{Results}

We analyzed the RT data as described previously. We excluded reading times that were more than 3 standard deviations from a participant's mean $(1.1 \%$ of the data). We also identified sentences that were associated with incorrect grammaticality responses and excluded the reading times for those sentences (6.5\% of the data).

Grammaticality judgments. We computed mean grammaticality judgments as a function of verb bias and span. The means appear in Table 5. The proportion of correct responses was high, and we found no differences as a function of verb bias or span $(F \mathrm{~s}<1)$. We also analyzed judgments to the filler sentences. The proportions of correct responses to these sentences were high $(M=$ $.91, .90$, and .87 for high span, medium span, and low span, respectively), and we found no span differences $(F \mathrm{~s}<1)$.

Reading-time analyses. We performed 2 (verb bias) $\times 3$ (span) ANOVAs on the mean reading times for each sentence region. The means appear in Table 6. Our analysis of reading times at the prepositional phrase yielded a reliable verb bias $\times$ span interaction $\left[F_{1}(2,45)=4.41, M S_{\mathrm{e}}=\right.$ $\left.1,547.89 ; F_{2}(2,177)=14.78, M S_{\mathrm{e}}=42,993.07\right]$. Reading times for high-span readers were slower in the past-tensebiased condition than they were in the past-participlebiased condition. This effect was reliable in the participant analysis $\left[F_{1}(2,45)=10.48, M S_{\mathrm{e}}=11,547, d=0.25\right]$, but not in the item analysis $\left[F_{2}(2,177)=1.73, M S_{\mathrm{e}}=\right.$ 42,993]. Medium-span and low-span readers showed no differences $(F \mathrm{~s}<1)$.

In the sentence-final region, we found a reliable effect of verb bias $\left[F_{1}(1,45)=38.48, M S_{\mathrm{e}}=4,305.72, d=0.28\right.$; $\left.F_{2}(1,177)=55.06, M S_{\mathrm{e}}=513,827\right]$. All participants read sentences in the past-tense-biased condition more slowly than they did in the past-participle-biased condition.

The results of this experiment replicate the patterns that we observed in Experiments 1A and 1B. Differences as a function of span occurred only at the prepositional phrase. The data from the grammaticality task indicated that both high-span and low-span readers thought that the ambiguous sentences were grammatical. These data are not direct evidence that low-span readers constructed the correct interpretation of the reduced-relative clause; they are consistent, however, with our other data showing no span differences in comprehension performance. Moreover, they are consistent with the lack of span differences on the rating and completion tasks.

The offline data that we presented in Experiment 1 (agent plausibility, theme plausibility, sentence completion, and grammaticality judgments) suggest that lowspan readers have knowledge about the sentences that they fail to use in their initial processing of the ambiguity. This is the same pattern that was reported by Pearlmutter and MacDonald (1995). They found span differences in reading times that were not reflected in readers' responses on their plausibility judgment tasks. In a series of regression analyses, they showed that high-span readers' times were predicted by the plausibility of the different interpretations of the sentences, whereas plausibility ratings did not predict low-span readers' performance. We conducted a

Table 5

Grammaticality Judgments (in Proportions Correct) As a Function of Verb Bias and Reading Span in Experiment 1C

\begin{tabular}{cccc}
\hline & \multicolumn{3}{c}{ Reading Span } \\
\cline { 2 - 4 } Verb Bias & High & Medium & Low \\
\hline Past tense & .94 & .92 & .91 \\
Past participle & .97 & .93 & .94 \\
\hline
\end{tabular}


Table 6

Reading Times (in Milliseconds) for the Prepositional-Phrase and Sentence-Final Regions As a Function of Verb Bias and Reading Span in Experiment 1C

\begin{tabular}{|c|c|c|c|c|c|c|}
\hline \multirow[b]{3}{*}{ Region Bias } & \multicolumn{6}{|c|}{ Reading Span } \\
\hline & \multicolumn{2}{|c|}{ High } & \multicolumn{2}{|c|}{ Medium } & \multicolumn{2}{|c|}{ Low } \\
\hline & $M$ & $S D$ & $M$ & $S D$ & $M$ & $S D$ \\
\hline \multicolumn{7}{|c|}{ Prepositional Phrase } \\
\hline Past tense & 1,099 & 342 & 990 & 378 & 1,099 & 352 \\
\hline Past participle & 1,013 & 340 & 989 & 369 & 1,089 & 374 \\
\hline \multicolumn{7}{|l|}{ Sentence Final } \\
\hline Past tense & 1,645 & 369 & 1,730 & 587 & 1,586 & 633 \\
\hline Past participle & 1,501 & 337 & 1,587 & 533 & 1,446 & 581 \\
\hline
\end{tabular}

similar set of analyses to examine readers' use of plausibility information in this study.

\section{Regression Analyses}

We conducted a series of regression analyses to examine readers' use of three sources of probabilistic information: verb frequency, contextual plausibility, and contextual predictability. These measures were used as predictors of the reading times that we collected in Experiments 1A-1C.

\section{Results and Discussion}

The reading-time data from participants in Experiments $1 \mathrm{~A}-1 \mathrm{C}$ were aggregated and averaged across participants to calculate the means for each of our 240 sentences (120 past-tense-biased and 120 past-participle-biased sentences). We conducted an initial regression analysis using the reading times for past-participle-biased sentences to predict the reading times for past-tense-biased sentences. This was done separately for the prepositional-phrase and sentence-final regions. The residuals were then used as the dependent variables in subsequent analyses. The size of the residuals indexes the amount of variance in the reading times to past-tense-biased sentences that was not explained by the reading times to past-participle-biased sentences.

The residuals were analyzed as a function of three types of predictors. The first was frequency information. We collected three frequency measures from the Penn Treebank Corpus for each of our past-tense-biased verbs. Base frequency was the natural logarithmic frequency of the base-verb form. Past-tense frequency was the frequency of the past-tense form relative to the total frequency of the -ed form. Past-participle frequency was the frequency of the past-participle form relative to the total frequency of the -ed form.

The second type of predictor was plausibility. We included two measures from the rating data in Experiment 1A: theme plausibility (i.e., the average rating of the initial noun phrase as a plausible theme of the verb in the relative clause) and agent plausibility (i.e., the average rating of the noun phrase as a plausible agent of the verb).

The third type of predictor was contextual predictability (Frisson, Rayner, \& Pickering, 2005). This was obtained from the sentence-completion data collected in Experiment 1A. Relative-clause continuation was calculated as the proportion of participants who completed the sentence fragments with a relative clause.

We included two additional predictor variables. One was transitivity: Each of the verbs in the past-tensebiased condition was coded as to whether it had both a transitive and an intransitive argument structure or had only a transitive one. The second predictor was the relation between the past-tense-biased and the pastparticiple-biased version of the sentences. Some of the sentences in the past-participle-biased condition were unambiguous in their reduced-relative interpretation (e.g., The chocolate eaten in the sun dripped down the child's arm). These sentences had verbs in the relative clause that were morphologically marked as past participle and had only transitive interpretations. Thus, we coded each past-tense-biased sentence as to whether it was paired with an ambiguous or unambiguous version in the past-participle-biased condition (ambiguity). We included this variable to determine whether the verbbias effect in Experiments $1 \mathrm{~A}-1 \mathrm{C}$ was due primarily to the ease with which readers were able to process the unambiguous sentences in the past-participle-biased condition.

Bivariate correlations among predictor variables. The correlations among the predictor variables appear in Table 7. Base frequency was correlated with past-tense frequency such that high-frequency verbs in their base form were also high-frequency in their past-tense form. In addition, past-tense frequency was negatively correlated with past-participle frequency such that the higher the past-tense frequency of the verb, the lower the past-participle frequency. Relative-clause continuation was correlated with three variables: past-participle frequency, theme plausibility, and agent plausibility. Sentences with high-frequency past-participle forms received more relative-clause contin-

Table 7

Bivariate Correlations Among the Predictor Variables

\begin{tabular}{lrrrrrrrr}
\hline & 1 & 2 & 3 & 4 & 5 & 6 & 7 & 8 \\
\hline 1. Ambiguity & & & & & & & \\
2. Base frequency & -.01 & & & & & & \\
3. Past-participle frequency & .02 & -.11 & & & & & \\
4. Past-tense frequency & -.01 & $.20^{*}$ & $-.56^{* *}$ & & & & \\
5. Theme plausibility & -.03 & .05 & .08 & .02 & & & \\
6. Agent plausibility & -.07 & -.03 & .09 & .00 & -.14 & & \\
7. Relative-clause continuation & -.01 & -.01 & $.20^{*}$ & .06 & $.21^{*}$ & $-.55^{* *}$ & \\
8. Transitivity & .03 & .05 & -.16 & .09 & .11 & -.14 & .05 \\
${ }^{*} p<.05$ (two-tailed). ${ }^{* *} p<.01$ (two-tailed). & & & & & &
\end{tabular}


uations than sentences with low-frequency past-participle forms. In addition, relative-clause continuations were produced more frequently when preceded by noun phrases that were plausible than when preceded by implausible themes and less frequently when preceded by noun phrases that were plausible than when preceded by implausible agents.

We computed a separate regression analysis for each span group. The results of these analyses appear in Table 8 .

High-span readers. Several variables were reliable predictors of the residuals for the prepositional phrase. The verb-bias effect was larger when a sentence in the past-tense-biased condition had a version in the pastparticiple-biased condition that was unambiguous. In addition, the verb-bias effect increased as a function of the plausibility of the noun phrase as an agent of the verb in the relative clause. Reductions in the verb-bias effect were associated with contextual predictability and theme plausibility. The effect decreased when a relative-clause continuation was likely and when the noun phrase was a plausible theme of the verb.

The influence of the predictor variables was smaller in the sentence-final region than at the prepositional phrase; however, the patterns were similar. Contextual predictability and agent plausibility were marginally related to the verbbias effect, as they were for the prepositional phrase. Theme plausibility remained a strong predictor of the effect.

Medium-span readers. Our regression analysis of the residuals for the prepositional phrase yielded a reliable effect of ambiguity. The residuals were larger when the pasttense-biased sentences were paired with unambiguous sentences than when they were paired with biased ones. We found marginal effects of contextual predictability and agent plausibility. These effects were in the same direction as those exhibited by the high-span readers.

The pattern of reliable effects was similar in our analysis of the residuals in the sentence-final region. Ambiguity and relative-clause continuation had the same effect as they did in the prepositional phrase; however, agent plausibility was no longer reliable.

Low-span readers. Low-span readers showed a marginal effect of ambiguity at the prepositional phrase. The residuals were diminished somewhat when a sentence in the past-tense-biased condition was paired with an unambiguous version. This effect was reliable in our analysis of the residuals in the sentence-final region. In addition, we found a reliable effect of contextual predictability. The verb-bias effect decreased as the proportion of relative-clause continuations increased. We found no effects of plausibility at either the prepositional phrase or the sentence-final region.

These results reveal similarities and differences in high-span and low-span readers' processing of mainverb/reduced-relative ambiguities. Low-span readers, like high-span and medium-span readers, were sensitive to ambiguity. The verb-bias effect was larger when a pasttense-biased sentence was paired with an unambiguous version than when it was paired with a biased one. This suggests that all readers comprehended the reducedrelative structures with relative ease when the verb in the reduced relative was unambiguously past participle and had only a transitive argument structure. The primary difference between the high-span and low-span readers was their use of plausibility information. High-span readers were sensitive to the plausibility of the noun phrase as both the theme and the agent of the verb. In contrast, lowspan readers showed no plausibility effects.

Our results confirm those reported earlier by Pearlmutter and MacDonald (1995). Low-span readers had knowledge relevant to the plausibility of main-verb and reducedrelative interpretations of sentences, as demonstrated by their performance on the rating and sentence-completion tasks; however, they did not use this information during sentence comprehension. Our goal in the next experiment

Table 8

Regression Analyses of Reading Times in Experiment 1 As a Function of Frequency, Plausibility, Predictability, Ambiguity, and Transitivity

\begin{tabular}{|c|c|c|c|c|c|c|}
\hline \multirow[b]{3}{*}{ Region } & \multicolumn{6}{|c|}{ Reading Span } \\
\hline & \multicolumn{2}{|c|}{ High } & \multicolumn{2}{|c|}{ Medium } & \multicolumn{2}{|c|}{ Low } \\
\hline & $\beta$ & $t$ & $\beta$ & $t$ & $\beta$ & $t$ \\
\hline \multicolumn{7}{|l|}{ Prepositional Phrase } \\
\hline Base frequency & -.01 & -0.14 & .04 & 0.48 & -.08 & -0.82 \\
\hline Past-participle frequency & .04 & 0.52 & -.08 & -0.67 & -.04 & -0.39 \\
\hline Past-tense frequency & -.01 & -0.11 & -.15 & -1.34 & -.16 & -1.42 \\
\hline Ambiguity & .20 & $2.92^{* *}$ & .34 & $3.19^{* *}$ & .17 & $1.72^{\dagger}$ \\
\hline $\mathrm{RC}$ continuation & -.24 & $-3.06^{* *}$ & -.19 & $-1.72^{\dagger}$ & .03 & 0.28 \\
\hline Theme plausibility & -.54 & $-7.82^{* *}$ & .05 & 0.49 & -.01 & -0.05 \\
\hline Agent plausibility & .34 & $4.20^{* *}$ & .17 & $1.75^{\dagger}$ & -.02 & -0.15 \\
\hline Transitivity & .01 & 0.13 & .07 & 0.85 & -.00 & -0.00 \\
\hline \multicolumn{7}{|l|}{ Sentence Final } \\
\hline Base frequency & .06 & 0.77 & -.00 & -0.01 & -.02 & -0.27 \\
\hline Past-participle frequency & .09 & 0.86 & .15 & 1.42 & .12 & 1.15 \\
\hline Past-tense frequency & .05 & 0.53 & .12 & 1.11 & .09 & 0.82 \\
\hline Ambiguity & .07 & 0.83 & .36 & $4.04^{* *}$ & .37 & $4.08^{* *}$ \\
\hline $\mathrm{RC}$ continuation & -.18 & $-1.86^{\dagger}$ & -.19 & $-1.84^{\dagger}$ & -.22 & $-2.14^{*}$ \\
\hline Theme plausibility & -.43 & $-4.95^{* *}$ & -.15 & -1.43 & -.04 & -0.46 \\
\hline Agent plausibility & .17 & $1.66^{\dagger}$ & -.00 & -0.04 & .14 & 1.37 \\
\hline Transitivity & -.02 & -0.12 & -.05 & -0.52 & -.00 & -0.01 \\
\hline
\end{tabular}


was to examine whether repeated exposure to main-verb/ reduced-relative ambiguities would encourage low-span readers to use knowledge that they otherwise fail to apply.

\section{EXPERIMENT 2}

Why do low-span readers fail to use their knowledge about the plausibility of main-verb and reduced-relative interpretations when processing ambiguous sentences? We examined two possibilities in this experiment. The first is that low-level processes, such as word recognition, are sufficiently difficult for low-span readers, so that they lack the resources to use contextual information during processing. This possibility is consistent with claims from Perfetti's (1985) verbal efficiency theory. According to his theory, most comprehension problems are secondary to slow and inefficient word recognition. Poor word-recognition processes consume working memory resources that would otherwise be used for the execution of higher level comprehension processes.

A second possibility is that low-span readers have knowledge about the probabilistic constraints that are relevant in ambiguity resolution, but these constraints are represented in memory somewhat weakly. This possibility is consistent with Perfetti and Hart's (2001) lexical-quality hypothesis and with the distributed-learning model of working memory limitations (MacDonald \& Christiansen, 2002). Perfetti, Wlotko, and Hart (2005) argued that good comprehenders, unlike poor ones, have strong episodic memory traces for words - traces that contain phonological, orthographic, and meaning information. Comprehension processes that depend on high-quality lexical representations-such as syntactic analysis - are negatively affected when low-quality representations are retrieved. According to MacDonald and Christiansen, good comprehenders, unlike poor ones, can use probabilistic constraints rapidly during reading because they have often done so in the past. Thus, poor comprehenders, including low-span readers, may need stronger contextual cues or more time to activate relevant knowledge during comprehension than do good readers.

In the present experiment, we examined readers' sensitivity to plausibility after being exposed repeatedly to main-verb/reduced-relative sentences. We constructed the materials to highlight one of the important cues in syntactic ambiguity resolution-animacy. The reducedrelative interpretation is more plausible in the context of an inanimate noun than in the context of an animate one. In Experiments 1A-1C, the importance of animacy in predicting the reduced-relative interpretation was not salient because all our filler sentences also began with inanimate noun phrases.

We recruited high-span and low-span readers from the students who had participated in Experiment 1A. These readers were exposed to a large set of sentences across 10 training sessions. All the sentences contained main-verb/ reduced-relative ambiguities, like those in Experiment 1. Half the sentences resolved to a reduced-relative interpretation and the other half resolved to a main-verb interpretation. Reduced-relative interpretations always appeared in the context of inanimate noun phrases; main-verb inter- pretations always appeared in the context of animate noun phrases. Thus, animacy was $100 \%$ predictive of structure in the context of this experiment.

We conducted the experiment in two phases. The first was an exposure phase in which readers were exposed repeatedly to sentences with main-verb/reduced-relative ambiguities. We manipulated the type of exposure in a within-participants design. We used the past-tense-biased sentences from Experiment 1 and randomly assigned the sentences to three item conditions. In the consistentexposure condition, participants received the sentences repeatedly and the sentences were identical across exposure sessions. In the varied-exposure condition, we retained the verb in the relative clause and wrote new sentences for the exposure phase. We placed the verbs from the test list into the reduced-relative syntactic frame and then changed the sentence context such that it varied across exposure sessions (e.g., The button ripped from the shirt fell to the ground, The page ripped from the book was thrown in the trash). In the unexposed condition, the sentences were not presented during the exposure sessions; they were presented only during the test phase. The second phase was an exact replication of Experiment 1A (test phase).

We examined readers' performance during the test phase as a function of exposure condition. Previous research on repeated reading has shown that reading time decreases when sentences are the same at first and second readings. The effect is due to perceptual fluency (repetition effects) and improvements in lexical access (Raney, 2003; Raney \& Rayner, 1995). In addition, readers are more likely to execute high-level comprehension processes, such as inference generation, during rereading than during initial reading (Bourassa, Levy, Dowin, \& Casey, 1998; Millis \& King, 2001; Millis, Simon, \& tenBroek, 1998; Raney, 1993). If low-span readers fail to use plausibility information because low-level processes, such as word recognition, are too resource consuming, then repeated reading should ease the difficulty associated with the execution of these processes. The effect of rereading, however, should be restricted to sentences in the consistent-exposure condition because this is the condition in which the same sentences appeared at exposure and at test. Thus, low-span readers should show slower reading times at the prepositional phrase for sentences in the past-tense-biased condition than for those in the past-participle-biased condition, but only for sentences that are in the consistent-exposure condition.

The other possibility is that low-span readers represent plausibility information more weakly than do high-span readers; thus, repeated exposure to the sentences should improve their ability to use probabilistic information during rereading, as predicted by the distributed-learning model (MacDonald \& Christiansen, 2002). Although we highlighted animacy as a cue in this experiment, rereading is likely to make other cues-such as verb frequency, structure frequency, and the conditional probability of animacy and frequency - salient as well. If repeated exposure to main-verb/reduced-relative ambiguities during exposure alerts low-span readers to cues that are important in resolving the ambiguities, then they may consider the reduced-relative interpretation whenever they encounter the ambiguity. If so, then low-span readers should show 
the ambiguity effect in all exposure conditions - that is, reading times for past-tense-biased sentences should be slower than those for past-participle-biased sentences.

We also conducted a set of regression analyses, as we did in Experiment 1. According to the distributed-learning model, exposure to main-verb/reduced-relative sentences should increase low-span readers' use of plausibility information. Low-span readers should show an ambiguity effect that increases as a function of agent plausibility and decreases as a function of theme plausibility.

\section{Method}

Participants. We recruited 10 high-span and 10 low-span readers from the students in Experiment 1A. They participated in 11 sessions and were paid $\$ 5 / \mathrm{h}$.

Materials. We created a material set for each exposure session (10 sessions for a total of 10 lists). We began with the 120 pasttense-biased sentences used in Experiment 1A. Each of the sentences was randomly assigned to one of three exposure conditions. In the consistent-exposure condition $(n=40)$, the sentences were unchanged and were identical across lists. In the varied-exposure condition $(n=40)$, we selected the verbs from the original pasttense-biased sentences in Experiment 1A and wrote new sentence contexts. Each verb appeared repeatedly across lists but had a different sentence context in each list (e.g., The ball tossed at the basket hit the rim, The blanket tossed in the laundry was very dirty). Finally, sentences in the no-exposure condition $(n=40)$ were not presented during the exposure phase; they appeared only in the test phase. Thus, each list in the exposure phase contained 80 past-tensebiased sentences: 40 in the consistent-exposure condition and 40 in the inconsistent-exposure condition. The remaining 40 past-tensebiased sentences appeared only in the test phase.

We also created a set of filler sentences, all of which resolved to a main-verb interpretation. These included the same verbs used in the consistent-exposure and varied-exposure conditions, but had new sentence frames. These sentences began with an animate noun phrase, followed by the ambiguous verb and then a direct object (e.g., The dog tossed the ball into the air). The direct object immediately followed the verb in order to preserve information about its transitive argument structure. In summary, animacy was completely predictive of the syntactic role of the verbs in all of the exposure materials. Verbs that followed inanimate noun phrases introduced a reduced-relative clause, and those that followed animate noun phrases were the main verb of the sentence.

We included a set of test questions in the exposure materials to encourage comprehension of the sentences. The questions followed only those sentences that resolved to a main-verb interpretation and were similar to those used in Experiment 1A (48 true/false paraphrases). Half the questions were false, and half were true.

The exposure sessions were followed by a test session. The materials in the test session were the same as those used in Experiment 1A. The test sentences included the 40 past-tense-biased sentences from the consistent-exposure condition, 40 past-tense-biased sentences from the varied-exposure condition, and 40 past-tense-biased sentences with no exposure. The test list also included the 120 past-participlebiased sentences and the filler sentences from Experiment 1A.

Procedure. Participants received 10 exposure sessions, 2 sessions/ week for a total of 5 weeks. Each session lasted for about an hour. The sentences were presented using the moving-window paradigm, as described in Experiment 1A. The last exposure session was followed 3 to 4 days later by the test session. The procedure for the test session was identical to that used in Experiment 1A.

\section{Results and Discussion}

We calculated the mean reading times for two regions of the sentence, as described in Experiment 1. Reading times that were more than 3 standard deviations from a participant's mean were excluded from the analyses $(1.3 \%$ of the data). The means for Experiment 2 appear in Table 9. We compared the performance of participants in Experiment 2 after they received exposure to the verbs (posttest) to their performance in Experiment 1A prior to exposure (pretest). We conducted 2 (verb bias) $\times 2$ (span) $\times 2$ (test) $\times 3$ (exposure) ANOVAs on the reading times from the two experiments.

Our analyses of the data at the prepositional phrase yielded several reliable main effects and interactions. We report here the critical four-way interaction between test, verb bias, span, and exposure; this interaction was reliable in both the participant and item analyses $\left[F_{1}(2,36)=\right.$ $\left.3.87, M S_{\mathrm{e}}=27,421 ; F_{2}(2,56)=9.33, M S_{\mathrm{e}}=57,667\right]$. The results for the pretest condition were similar to those reported in Experiment 1A. High-span readers showed an effect of verb bias, responding slower to sentences in the past-tense-biased condition than they did to sentences in the past-participle-biased condition $\left[F_{1}(1,18)=\right.$ $4.47, M S_{\mathrm{e}}=2,282, d=0.15 ; F_{2}(1,39)=88.38, M S_{\mathrm{e}}=$ 34,588], whereas low-span readers showed no effect of verb bias $\left[F_{1}<1 ; F_{2}(1,39)=1.36, M S_{\mathrm{e}}=67,790, p=\right.$ .26]. We predicted no effect of exposure condition at pretest, since the data were collected before the exposure phase. As expected, exposure condition was not significant $(F \mathrm{~s}<1)$.

With respect to the posttest condition, we found no reliable effects of span, unlike the pattern that we saw at pretest. In the consistent and varied conditions, we found a verb-bias effect that was reliable in the item analysis $\left[F_{2}(1,39)=24.11, M S_{\mathrm{e}}=24,116\right.$ (consistent); and

Table 9

Reading Times (in Milliseconds) for the Prepositional-Phrase and Sentence-Final Regions As a Function of Test, Reading Span, Verb Bias, and Exposure Conditions in Experiment 2

\begin{tabular}{|c|c|c|c|c|c|c|c|c|c|c|c|c|}
\hline \multirow[b]{3}{*}{ Region Bias } & \multicolumn{6}{|c|}{ Pretest Exposure } & \multicolumn{6}{|c|}{ Posttest Exposure } \\
\hline & \multicolumn{2}{|c|}{ Consistent } & \multicolumn{2}{|c|}{ Varied } & \multicolumn{2}{|c|}{ No Exposure } & \multicolumn{2}{|c|}{ Consistent } & \multicolumn{2}{|c|}{ Varied } & \multicolumn{2}{|c|}{ No Exposure } \\
\hline & $\begin{array}{l}\text { High } \\
\text { Span }\end{array}$ & $\begin{array}{l}\text { Low } \\
\text { Span }\end{array}$ & $\begin{array}{l}\text { High } \\
\text { Span }\end{array}$ & $\begin{array}{l}\text { Low } \\
\text { Span }\end{array}$ & $\begin{array}{l}\text { High } \\
\text { Span }\end{array}$ & $\begin{array}{l}\text { Low } \\
\text { Span }\end{array}$ & $\begin{array}{l}\text { High } \\
\text { Span }\end{array}$ & $\begin{array}{l}\text { Low } \\
\text { Span }\end{array}$ & $\begin{array}{l}\text { High } \\
\text { Span }\end{array}$ & $\begin{array}{l}\text { Low } \\
\text { Span }\end{array}$ & $\begin{array}{l}\text { High } \\
\text { Span }\end{array}$ & $\begin{array}{l}\text { Low } \\
\text { Span }\end{array}$ \\
\hline \multicolumn{13}{|c|}{ Prepositional Phrase } \\
\hline Past tense & 1,284 & 1,031 & 1,269 & 1,037 & 1,271 & 1,040 & 450 & 576 & 433 & 700 & 715 & 852 \\
\hline Past participle & 1,180 & 1,026 & 1,175 & 1,022 & 1,193 & 1,022 & 414 & 491 & 374 & 529 & 475 & 537 \\
\hline \multicolumn{13}{|l|}{ Sentence Final } \\
\hline Past tense & 1,496 & 1,575 & 1,491 & 1,567 & 1,489 & 1,584 & 637 & 900 & 618 & 869 & 854 & 817 \\
\hline Past participle & 1,350 & 1,432 & 1,346 & 1,431 & 1,339 & 1,447 & 545 & 729 & 518 & 689 & 627 & 665 \\
\hline
\end{tabular}


$F_{2}(1,39)=10.23, M S_{\mathrm{e}}=9,686$ (varied)] but not in the participant analysis $\left[F_{1}(1,19)=1.50, M S_{\mathrm{e}}=103,411\right.$, $p=.24, d=0.19$ (consistent); and $F_{1}(1,19)=1.86$, $M S_{\mathrm{e}}=16,700, p=.19, d=0.32$ (varied)]. In the noexposure condition, the verb-bias effect was reliable in both analyses $\left[F_{1}(1,19)=6.17, M S_{\mathrm{e}}=112,031, d=0.69\right.$; $\left.F_{2}(1,39)=48.13, M S_{\mathrm{e}}=38,725\right]$. Thus, readers showed a larger verb-bias effect for unexposed sentences than they did for sentences in the consistent or varied conditions.

Our analyses of reading times in sentence-final region also yielded several reliable main effects and interactions. The critical four-way interaction between test, verb bias, span, and exposure was not reliable $(F \mathrm{~s}<1)$; however, we did find a reliable interaction between test, verb bias, and exposure $\left[F_{1}(1,18)=28.79, M S_{\mathrm{e}}=15,650 ; F_{2}(1,39)=\right.$ $\left.10.01, M S_{\mathrm{e}}=37,718\right]$. The results for the pretest condition were similar to those reported in Experiment 1A. Participants showed a verb-bias effect $\left[F_{1}(1,18)=64.64, M S_{\mathrm{e}}=\right.$ $\left.34,992, d=0.30 ; F_{2}(1,39)=12.34, M S_{\mathrm{e}}=30,718\right]$. The verb-bias effect did not differ as a function of span $\left(F_{\mathrm{S}}<1\right)$. Once again, we found no effect of exposure in the pretest data $(F \mathrm{~S}<1)$.

With respect to the posttest data, we found that sentences in the consistent and varied conditions were read more slowly in the past-tense-biased condition than they were in the past-participle-biased condition. The effect in the consistent condition was reliable in the item analysis $\left[F_{2}(1,39)=7.71, M S_{\mathrm{e}}=43,250\right]$ but not in the participant analysis $\left[F_{1}(1,19)=1.02, M S_{\mathrm{e}}=169,052, p=.33, d=\right.$ $0.30]$. The effect in the varied condition was marginally reliable in the item analysis $\left[F_{2}(1,39)=2.71, M S_{\mathrm{e}}=49,127\right.$, $p=.10]$ but not in the participant analysis $\left[F_{1}(1,19)=\right.$ $\left.1.46, M S_{\mathrm{e}}=55,026, p=.24, d=0.32\right]$. Finally, in the noexposure condition, both high-span and low-span readers were reliably slower to read past-tense-biased than they were to read past-participle-biased sentences $\left[F_{1}(1,19)=\right.$ $7.44, M S_{\mathrm{e}}=81,360, d=0.44 ; F_{2}(1,39)=19.08, M S_{\mathrm{e}}=$ $59,437]$. Once again, readers showed a larger verb-bias effect for unexposed sentences than they did for sentences in the consistent or varied conditions.

\section{Regression Analyses}

We did not have enough items to conduct separate regression analyses in each exposure condition. Therefore, we collapsed across exposure and analyzed the data as we did in Experiment 1. The results appear in Table 10.

In our analysis of data at the prepositional phrase, we found results for the high-span readers that were similar to the pattern that we saw in Experiment 1, although the effects were somewhat smaller. The verb-bias effect increased as a function of agent plausibility and decreased as a function of theme plausibility. The primary difference between this analysis and the one reported in Experiment 1 concerned the performance of the low-span readers. We found reliable effects of theme plausibility and agent plausibility that we did not see previously.

In the sentence-final region, we found an overall reduction in the size of the effects, both when comparing the results to the prepositional phrase and when comparing the results to those reported in Experiment 1.
Table 10

Regression Analyses of Reading Times in Experiment 2 As a Function of Frequency, Plausibility, Predictability, Ambiguity, and Transitivity

\begin{tabular}{|c|c|c|c|c|}
\hline \multirow[b]{3}{*}{ Region } & \multicolumn{4}{|c|}{ Reading Span } \\
\hline & \multicolumn{2}{|c|}{ High } & \multicolumn{2}{|c|}{ Low } \\
\hline & $\beta$ & $t$ & $\beta$ & $t$ \\
\hline \multicolumn{5}{|l|}{ Prepositional Phrase } \\
\hline Base frequency & .08 & 0.96 & -.07 & -0.79 \\
\hline Past-participle frequency & -.07 & -0.74 & -.03 & -0.30 \\
\hline Past-tense frequency & -.09 & -0.88 & -.05 & -0.51 \\
\hline Ambiguity & .13 & 1.61 & .15 & $1.75^{\dagger}$ \\
\hline $\mathrm{RC}$ continuation & -.12 & -1.40 & -.11 & -1.09 \\
\hline Theme plausibility & -.26 & $-2.92^{* *}$ & -.21 & $-2.29^{*}$ \\
\hline Agent plausibility & .43 & $4.31^{* *}$ & .37 & $3.61^{* * *}$ \\
\hline Transitivity & -.09 & -0.96 & .08 & 0.99 \\
\hline \multicolumn{5}{|l|}{ Sentence Final } \\
\hline Base frequency & .05 & 0.49 & .01 & 0.10 \\
\hline Past-participle frequency & -.01 & -0.07 & .03 & 0.27 \\
\hline Past-tense frequency & -.09 & -0.75 & .08 & 0.87 \\
\hline Ambiguity & .05 & 0.56 & .12 & 1.07 \\
\hline $\mathrm{RC}$ continuation & -.03 & -0.29 & .01 & 0.11 \\
\hline Theme plausibility & -.06 & -0.50 & -.15 & -1.31 \\
\hline Agent plausibility & .19 & 1.59 & .22 & $1.87^{\dagger}$ \\
\hline Transitivity & .04 & 0.39 & .04 & 0.38 \\
\hline
\end{tabular}

We found only one effect that approached significance. Low-span readers showed an effect of agent plausibility; the verb-bias effect increased as a function of the plausibility of the initial noun phrase as an agent of the verb. Thus, the primary effect of exposure on reading times in the sentence-final region was to diminish the amount of explained variance.

\section{GENERAL DISCUSSION}

Our goal in this study was to examine how individual differences in working memory capacity, as assessed by reading span, affect syntactic processing. Previous research on this topic has yielded inconsistent results. Some studies have found that low-span readers process syntactically complex sentences more slowly than do high-span readers (King \& Just, 1991); some have found the opposite pattern (MacDonald et al., 1992; Pearlmutter \& MacDonald, 1995); and still others have found no systematic relation between span and syntactic parsing (Caplan \& Waters, 1999; Clifton et al., 2003; Waters \& Caplan, 1996).

In Experiment 1, we found that high-span readers processed syntactic ambiguities differently than did low-span readers. Reading times at the prepositional phrase were slower in past-tense-biased sentences than they were in past-participle-biased sentences, but only among highspan readers. This suggests that these readers were sensitive to the possibility that the preferred main-verb interpretation might not be correct. Low-span readers, in contrast, showed an ambiguity effect at the end of the sentence, only after the main verb of the sentence had been encountered. This pattern of results was consistent across three experiments and was not affected by the task that was associated with reading. We found the same results when comprehension questions were directed at the experimental sentences as we did when the questions were directed at the filler sen- 
tences. Moreover, we found the same results when readers made grammaticality judgments about the sentences.

One particularly interesting result in Experiment 1 was the contrast between low-span readers' performance on the offline tasks (sentence completion, ratings, and grammaticality judgments) and their pattern of reading times. High-span and low-span readers performed similarly on the offline tasks. Thus, low-span readers had knowledge about the probabilistic constraints that was relevant to the main-verb/reduced relative ambiguities, but this knowledge did not affect their reading behavior.

Our regression analyses in Experiment 1 confirmed that low-span readers did not use the same information during comprehension as did high-span readers. High-span readers' performance was affected by both plausibility and predictability. The verb-bias effect increased as a function of agent plausibility. Theme plausibility and predictability affected reading times in the opposite direction. The ambiguity effect decreased as a function of theme plausibility and relative-clause predictability. Low-span readers, in contrast, were affected only by morphological information in the past-participle-biased condition. This condition included some sentences that were unambiguous in their reduced-relative interpretation; the verbs in the relative clause were morphologically marked as past participle (e.g., eaten, bitten) and had only a transitive argument structure. When a past-tense-biased sentence was paired with an unambiguous version, low-span readers showed an ambiguity effect. This suggests that they processed the unambiguous versions with relative ease.

Low-span readers' failure to use knowledge that they clearly possess during reading is consistent with previous findings by Pearlmutter and MacDonald (1995), who collected plausibility ratings of different syntactic interpretations from high-span and low-span readers and found no differences in the information that the groups supplied. Nonetheless, they found that the two groups showed differences in reading times for main-verb/reduced-relative ambiguities that resolved to main-verb interpretations. In addition, Pearlmutter and MacDonald conducted regression analyses similar to those that we reported. They found that high-span readers' reading times were influenced by their knowledge about plausibility, whereas low-span readers showed no plausibility effects.

Studies of syntactic processing are not the only ones to show that poor comprehenders, including low-span readers, fail to use knowledge during reading that they use in performing offline tasks. Long and her colleagues have shown that poor comprehenders (1) fail to make thematic inferences during reading even though they can generate the inferences in question-answering tasks (Long et al., 1994, 1997), (2) fail to use knowledge about the implicit causality of verbs during pronoun resolution even though they use the knowledge when asked explicitly about the referents of pronouns (Long \& De Ley, 2000), and (3) are insensitive to contradictions during story reading even though they can identify the contradiction when asked explicitly to do so (Long \& Chong, 2001).

Our goal in Experiment 2 was to investigate why lowspan readers fail to use plausibility knowledge when they process main-verb/reduced-relative ambiguities. We examined two possibilities: (1) They fail to do so because low-level processes are too resource consuming for higher level processes to be executed or (2) their knowledge is represented too weakly to be activated as strongly or as quickly as needed. Our findings in Experiment 2 are consistent with the latter explanation. Low-span readers were given repeated exposure to main-verb/reduced-relative ambiguities in an experimental context that highlighted knowledge relevant to the ambiguity (animacy). Subsequently, they showed an ambiguity effect at the prepositional phrase that they did not show in Experiment 1. This occurred even for sentences that did not appear during exposure. One caveat to this conclusion is that the sentences in the no-exposure condition were not novel. The readers were exposed to them in Experiment 1A. If a single exposure 6 weeks earlier was sufficient to provide a rereading benefit, then low-span readers may have had more resources available to process the sentences in Experiment 2 than they did in Experiment 1A. One aspect of our data, however, argues against this possibility. The ambiguity effect in the no-exposure condition was larger than the effect in the consistent and varied conditions. We would have expected the opposite pattern if the primary benefit of rereading was to automate the low-level processes that operate during comprehension.

Our findings in Experiment 2 are consistent with previous research on the benefits of rereading. Most research has found that rereading enhances both the perceptual processes involved in reading and higher level comprehension processes related to deriving sentence meaning. Rereading improves comprehension even when the perceptual characteristics of a text, such as font, are changed between initial and subsequent readings. Our findings are also consistent with recent research examining the benefits of rereading on processing novel syntactic structures. Kaschak (2006) and Kaschak and Glenberg (2004) examined readers' acquisition of the "needs" construction (e.g., The meal needs cooked), a structure that appears among speakers in the northern midland region of the United States, including Pennsylvania, Ohio, Indiana, Illinois, and some of Iowa. Participants in the upper Midwest region (Wisconsin and Minnesota) were exposed to this construction in a series of conversations. They learned the new construction rapidly and their understanding of the structure generalized to new contexts, when the construction was used with a different verb (e.g., The dog wants walked).

Individual differences in syntactic parsing are interesting, in part, because they have implications for understanding the nature of working memory limitations and how these limitations influence language comprehension. We described three different views of working memory limitations: one that attributes variation in working memory to the amount of activation available for computation and storage (capacity theory), one that emphasizes the roles of knowledge and practice in language comprehension (distributed-learning model), and one that argues for a pool of resources that is dedicated to sentence interpretation and does not vary across individuals (separate sentence interpretation resource theory). Our results are clearly in- 
consistent with the predictions from the separate sentence interpretation resource theory. We found variation in readers' interpretation of the syntactic ambiguities, even in the absence of questions that highlighted the nature of the experimental materials. Moreover, we found that exposure enhanced the ambiguity effect in low-span readers.

The capacity theory and the distributed-learning models predict the pattern of reading times that we found in Experiment 1 . The results of Experiment 2 are predicted by the distributed-learning model but require additional assumptions with respect to capacity theory. The distributedlearning model attributes span differences in processing syntactic ambiguities to low-span readers' inexperience with these structures. Thus, repeated exposure should minimize span differences. This is what we found in Experiment 2. Moreover, the effect of exposure was to enhance low-span readers' use of knowledge about the plausibility of the various interpretations. Capacity theory, however, attributes span differences to variation in the amount of activation that is available to the working memory system. In order to account for the effects of exposure, we would need to assume that repeated exposure to the main-verb/ reduced-relative ambiguities increased the amount of activation available to low-span readers. Capacity theory does not currently have a mechanism that changes the system's total activation as a function of experience.

In summary, our results suggest that sentence understanding involves a complex interaction of reader characteristics and sentence properties. Low-span readers do not use plausibility information during comprehension of main-verb/reduced-relative ambiguities even though they clearly possess this knowledge. Our results suggest that their failure to use relevant knowledge is not due to fixed capacity limitations. Their performance is similar to that of high-span readers when the experimental context encourages their use of the knowledge. Thus, our data show that the relation between working memory and syntactic parsing is relatively malleable; it depends critically on the experience of individuals in the experiment as well as on the properties of the stimuli.

\section{AUTHOR NOTE}

The research reported here was supported by National Institutes of Health Grant NICHD 1 R01 HD48914-1A2. Correspondence concerning this article should be addressed to D. L. Long, Department of Psychology, One Shields Avenue, University of California, Davis, CA 95616 (e-mail: dllong@ucdavis.edu).

\section{REFERENCES}

Bell, L. C., \& Perfetti, C. A. (1994). Reading skill: Some adult comparisons. Journal of Educational Psychology, 86, 244-255.

BEver, T. G. (1970). The cognitive basis for linguistic structures. In J. R. Hayes (Ed.), Cognition and the development of language (pp. 279-360). New York: Wiley.

Bourassa, D. C., Levy, B. A., Dowin, S., \& Casey, A. (1998). Transfer effects across contextual and linguistic boundaries: Evidence from poor readers. Journal of Experimental Child Psychology, 71, 45-61.

CAPLAN, D., \& Waters, G. S. (1999). Verbal working memory and sentence comprehension. Behavioral \& Brain Sciences, 22, 77-126.

Clifton, C., Jr., Traxler, M. J., Mohamed, M. T., Williams, R. S., Morris, R. K., \& RAYNER, K. (2003). The use of thematic role information in parsing: Syntactic processing autonomy revisited. Journal of Memory \& Language, 49, 317-334.
Daneman, M., \& Carpenter, P. A. (1980). Individual differences in working memory and reading. Journal of Verbal Learning \& Verbal Behavior, 19, 450-466.

Frazier, L. (1987). Sentence processing: A tutorial review. In M. Coltheart (Ed.), Attention and performance XII: The psychology of reading (pp. 559-586). Hillsdale, NJ: Erlbaum.

Frazier, L., \& Clifton, C., JR. (1996). Construal. Cambridge, MA: MIT Press.

Frisson, S., RAYNer, K., \& Pickering, M. J. (2005). Effects of contextual predictability and transitional probability on eye movements during reading. Journal of Experimental Psychology: Learning, Memory, \& Cognition, 31, 862-877.

Gernsbacher, M. A., \& Faust, M. E. (1991). The mechanism of suppression: A component of general comprehension skill. Journal of Experimental Psychology: Learning, Memory, \& Cognition, 17, 245-262.

Gernsbacher, M. A., \& FAust, M. E. (1995). Skilled suppression. In F. N. Dempster \& C. J. Brainerd (Eds.), Interference and inhibition in cognition (pp. 295-327). San Diego: Academic Press.

Hare, M., Tanenhaus, M. K., \& McRae, K. (2007). Understanding and producing the reduced relative construction: Evidence from ratings, editing, and corpora. Journal of Memory \& Language, 56, 410-435.

Just, M. A., \& CARPEnTER, P. A. (1992). A capacity theory of comprehension: Individual differences in working memory. Psychological Review, 99, 122-149.

KaschaK, M. P. (2006). What this construction needs is generalized. Memory \& Cognition, 34, 368-379.

Kaschak, M. P., \& Glenberg, A. M. (2004). This construction needs learned. Journal of Experimental Psychology: General, 133, 450-467.

KING, J., \& Just, M. A. (1991). Individual differences in syntactic processing: The role of working memory. Journal of Memory \& Language, 30, 580-602.

LONG, D. L., \& ChONG, J. L. (2001). Comprehension skill and global coherence: A paradoxical picture of poor comprehenders' abilities. Journal of Experimental Psychology: Learning, Memory, \& Cognition, 27, 1424-1429.

LONG, D. L., \& DE LEy, L. (2000). Implicit causality and discourse focus: The interaction of text and reader characteristics in pronoun resolution. Journal of Memory \& Language, 42, 545-570.

LoNG, D. L., Oppy, B. J., \& SeELy, M. R. (1994). Individual differences in the time course of inferential processing. Journal of Experimental Psychology: Learning, Memory, \& Cognition, 20, 1456-1470.

Long, D. L., Oppy, B. J., \& Seely, M. R. (1997). Individual differences in readers' sentence- and text-level representations. Journal of Memory \& Language, 36, 129-145.

MacDonald, M. C., \& Christiansen, M. H. (2002). Reassessing working memory: Comment on Just and Carpenter (1992) and Waters and Caplan (1996). Psychological Review, 109, 35-54.

MacDonald, M. C., Just, M. A., \& Carpenter, P. A. (1992). Working memory constraints on the processing of syntactic ambiguity. Cognitive Psychology, 24, 56-98.

MacDonald, M. C., Pearlmutter, N. J., \& Seidenberg, M. S. (1994). Lexical nature of syntactic ambiguity resolution. Psychological Review, 101, 676-703.

Marcus, M. P., Marcinkiewicz, M. A., \& Santorini, B. (1993). Building a large annotated corpus of English: The Penn Treebank. Computational Linguistics, 19, 313-330.

McRae, K., Ferretti, T. R., \& Amyote, L. (1997). Thematic roles as verbspecific concepts. Language \& Cognitive Processes, 12, 137-176.

McRae, K., Spivey-Knowlton, M. J., \& Tanenhaus, M. K. (1998). Modeling the influence of thematic fit (and other constraints) in online sentence comprehension. Journal of Memory \& Language, 38, 283-312.

MiLLIS, K. K., \& KING, A. (2001). Rereading strategically: The influences of comprehension ability and a prior reading on the memory for expository text. Reading Psychology, 22, 41-65.

Millis, K. K., Simon, S., \& tenBroeK, N. S. (1998). Resource allocation during the rereading of scientific texts. Memory \& Cognition, 26, 232-246.

Pearlmutter, N. J., \& MacDonald, M. C. (1995). Individual differences and probabilistic constraints in syntactic ambiguity resolution. Journal of Memory \& Language, 34, 521-542. 
Perfetti, C. A. (1985). Reading ability. New York: Oxford University Press.

Perfetti, C. A., \& Hart, L. (2001). The lexical basis of comprehension skill. In D. S. Gorfein (Ed.), On the consequences of meaning selection: Perspectives on resolving lexical ambiguity (pp. 67-86). Washington, DC: American Psychological Association.

Perfetti, C. A., Wlotko, E. W., \& Hart, L. A. (2005). Word learning and individual differences in word learning reflected in event-related potentials. Journal of Experimental Psychology: Learning, Memory, \& Cognition, 31, 1281-1292.

RANEY, G. E. (1993). Monitoring changes in cognitive load during reading: An event-related brain potential and reaction time analysis. Journal of Experimental Psychology: Learning, Memory, \& Cognition, 19, 51-69.

RANey, G. E. (2003). A context-dependent representation model for explaining text repetition effects. Psychonomic Bulletin \& Review, 10, 15-28.

RANey, G. E., \& RAYNer, K. (1995). Word frequency effects and eye movements during two readings of a text. Canadian Journal of Experimental Psychology, 49, 151-173.

Stanovich, K. E., \& WeSt, R. F. (1989). Exposure to print and orthographic processing. Reading Research Quarterly, 24, 402-433.

Tabossi, P., Spivey-Knowlton, M. J., McRae, K., \& Tanenhaus, M. K.
(1994). Semantic effects on syntactic ambiguity resolution: Evidence for a constraint-based resolution process. In C. Umiltà \& M. Moscovitch (Eds.), Attention and performance XV: Conscious and nonconscious information processing (pp. 589-615). Cambridge, MA: MIT Press.

Trueswell, J. C. (1996). The role of lexical frequency in syntactic ambiguity resolution. Journal of Memory \& Language, 35, 566-585.

Trueswell, J. C., Tanenhaus, M. K., \& Garnsey, S. M. (1994). Semantic influences on parsing: Use of thematic role information in syntactic ambiguity resolution. Journal of Memory \& Language, 33, 285-318.

Waters, G. S., \& CaPlan, D. (1996). Processing resource capacity and the comprehension of garden path sentences. Memory \& Cognition, 24, 342-355.

\section{NOTE}

1. Reading span is often calculated as set size - the largest set size for which a participant can recall all the sentence-final words-with fractions accrued for partially recalled sets. We chose to use the total number of sentence-final words recalled in order to obtain a greater range of reading-span scores. This enabled us to divide our participants evenly into three groups: high-span, medium-span, and low-span readers. However, we also calculated reading span as set size.

\section{APPENDIX \\ Test Sentences}

1. The first question asked/written on the exam was particularly difficult.

2. The revolution started/born in the city spread throughout the country.

3. The salad tossed/mixed for the party looked delicious.

4. The prayers said/spoken in the night offered comfort to the victims.

5. The tooth pulled/removed from his mouth was black with decay.

6. The climb attempted/accomplished in the winter cost several people their lives.

7. The fence jumped/erected before the event was clipped by the horse's hoof.

8. The knee scraped/wounded in the accident was treated with antibiotics.

9. The foot raked/hurt in the garden needed ten stitches.

10. The game watched/promoted on the television was exciting.

11. The bowl dropped/thrown in the kitchen broke into tiny pieces.

12. The ship sailed/sunk in the ocean carried several lifeboats.

13. The merchandise returned/distributed to the store was damaged.

14. The continents fused/linked during the earthquake drifted apart over time.

15. The dart blasted/blown from the tube hit the target.

16. The manuscript scrutinized/altered at the institute was thought to be a forgery.

17. The vase smashed/broken during the earthquake was irreplaceable.

18. The verses shouted/sung from the pulpit moved the congregation to tears.

19. The question answered/assigned in the class appeared on the final exam.

20. The details recalled/mistaken in the interview were carefully recorded.

21. The water poured/purified through the filter was clear and fresh.

22. The money flashed/invested in the casino earned the executive lavish privileges.

23. The park visited/illuminated in the evening was always crowded.

24. The pants ripped/torn in the game were ruined beyond repair.

25. The gift opened/given at the party was a lovely scarf.

26. The cheese smoked/aged in the cellar tasted wonderful.

27. The button yanked/detached from the shirt left a hole in the fabric.

28. The chemicals supervised/isolated in the warehouse were quite dangerous.

29. The meat savored/consumed in the jungle was the last of the explorer's supplies.

30. The crate moved/loaded in the attic was very heavy.

31. The skull cursed/shrunken in the ritual was used to ward off evil spirits.

32. The data pondered/calculated in the laboratory was inconclusive.

33. The metal struck/molded with the mallet was bent into the proper shape.

34. The seasonings sprinkled/shaken on the turkey complimented its natural flavors.

35. The car raced/driven down the freeway was chased by the highway patrol.

36. The money handed/transferred to the robbers was counterfeit.

37. The torture inflicted/administered at the jail was documented in the lawsuit.

38. The offer declined/related in the meeting was insufficient to cover the victim's losses.

39. The insult excused/forgiven during the argument was still not forgotten.

40. The cleansers kept/organized in the cabinet were poisonous. 


\section{APPENDIX (Continued)}

41. The vows improvised/sworn at the wedding left tears in everyone's eyes.

42. The chocolate licked/eaten in the sun dripped down the child's arm.

43. The vehicle dumped/deserted in the alley had been stripped of its valuable parts.

44. The convertible waxed/viewed at the showroom sparkled in the afternoon sun.

45. The milk spilled/processed at the plant was contaminated.

46. The portraits hung/honored in the museum were painted by an unknown artist.

47. The ransom demanded/financed after the kidnapping was paid in unmarked bills.

48. The words remembered/forgotten from the list were analyzed by the experimenter.

49. The prize claimed/chosen for the winner was an autographed football jersey.

50. The fabric stretched/woven for the blanket was soft and warm.

51. The boat gouged/stricken in the storm sank two miles from shore.

52. The city won/governed during the war obeyed a strict curfew.

53. The claim affirmed/justified during the trial resulted in a settlement offer.

54. The profit declared/determined at the session was grossly overstated.

55. The confession admitted/obtained in the interview was thrown out of court.

56. The mission chanced/undertaken in the spring yielded better than expected results.

57. The arrow pointed/oriented to the north told the hiker that he was not lost.

58. The wine tasted/experienced at the party was chosen carefully to complement the food.

59. The eggs cracked/beaten for the omelet were gathered earlier that morning.

60. The colors brushed/drawn on the canvas formed a beautiful stained glass effect.

61. The script sought/adapted for the movie was written by a well-known author.

62. The gown wanted/sewn for the ball had lace on the sleeves.

63. The building realized/rendered on the drawings would be beautiful when it was finished.

64. The flowers clipped/grown in the garden were used to decorate the table.

65. The clothing stiffened/frozen from the snow hung by the fire to dry.

66. The flour sifted/molded into the dough was measured carefully.

67. The information scanned/retrieved from the document was entered into the database.

68. The arm grabbed/injured during the wrestling match was black and blue.

69. The amount owed/taxed for the year was written on the invoice.

70. The protest provoked/muffled at the meeting resulted in a strike by the union.

71. The car thrust/accelerated into fourth gear stalled on the uphill slope.

72. The message preached/hidden in the passage was that good and evil are always at war.

73. The story believed/translated at the university was distributed over the Internet.

74. The piano wheeled/located on the stage was shipped from Europe.

75. The cheer cried/executed from the stands was heard throughout the stadium.

76. The problems envisioned/foreseen in the report can be avoided only if action is taken immediately.

77. The event entered/swum at the competition drew large crowds.

78. The relic snatched/stolen at the site was a priceless piece of history.

79. The items searched/limited at the border were all agricultural products.

80. The ball bounced/balanced on the racket was bright orange.

81. The tent wet/stored after the storm smelled musty and stale.

82. The water dripped/absorbed into the dough made it easier to knead.

83. The guns surrendered/taken during the arrest had been illegally obtained.

84. The shoes felt/seen under the bed were covered with dust.

85. The fixture snapped/screwed into the base was made of lightweight aluminum.

86. The cheek caressed/swollen after the injury was bruised and sore.

87. The glider plunged/flown from the building hit the ground before our eyes.

88. The books despised/forbidden at the church were burned in a special ceremony.

89. The jewels gathered/shown at the museum had belonged to an Egyptian pharaoh.

90. The report weighed/analyzed at the meeting influenced the board's decision.

91. The dispute decided/exposed at the trial cost both companies thousands of dollars.

92. The beverage liked/tested at the fair won first prize in its category.

93. The confirmation upheld/begun in the Senate was broadcast on cable news channels.

94. The traffic slowed/concentrated behind the accident stretched for miles.

95. The friendship betrayed/upset during the argument would be difficult to repair.

96. The message ended/omitted at the tone was from a solicitor.

97. The chairs straightened/seated at the table were covered in velvet.

98. The motion disregarded/sustained at the trial served as the basis for the prisoner's appeal.

99. The logic explained/constructed during the lecture was quite complicated.

100. The totals figured/based on erroneous data cost the company millions of dollars.

101. The seminar concluded/scheduled in the afternoon provided information about new cancer treatments.

102. The glass hurled/flung across the table was empty.

103. The examination failed/completed at the school would influence future funding.

104. The quotation lifted/gotten from the article was included to bolster his argument. 
APPENDIX (Continued)

105. The vehicle possessed/manned at the airbase was both fast and reliable.

106. The watch wound/worn in the shower stopped working.

107. The colors scrambled/integrated on the canvas created an unusual visual effect.

108. The trap gripped/sprung from the bottom was guaranteed to capture the snake.

109. The dissension sensed/illustrated at the meeting contributed to the company's low morale.

110. The decorating enjoyed/done at the event required hours of planning and work.

111. The procedure refused/rejected in the hospital was very dangerous.

112. The books counted/valued at the library were donated by a wealthy patron.

113. The apples shifted/fallen from the basket rolled across the floor.

114. The island reached/arisen over the sea was formed in an earthquake.

115. The attitude inferred/deduced from the article was that diplomacy would be more successful than military force.

116. The drugs managed/dispensed at the clinic kept his addiction under control.

117. The concerns confided/acknowledged during the session were surprisingly easy to resolve.

118. The apple chewed/bitten during the break had a worm in it.

119. The motion appealed/denied at the trial was important to the defendant's case.

120. The rock clamped/concealed in his fist was shiny and smooth.

Note-Verbs appear in the order past-tense biased/past-participle biased.

(Manuscript received April 10, 2006;

revision accepted for publication August 19, 2007.) 